\title{
Processamento e estudo da estabilidade de pasta de pequi (Caryocar brasiliense)
}

\author{
Processing and stability study of pequi paste (Caryocar brasiliense)
}

\author{
Aroldo ARÉVALO-PINEDO ${ }^{1 *}$, Vinicius Borges Vieira MACIEL ${ }^{1}$, Kallyana Moraes CARVALHO ${ }^{1}$, \\ Ana Flavia Santos COELHO ${ }^{1}$, Abraham Damian GIRALDO-ZUÑIGA ${ }^{1}$, \\ Zilda Doratiotto de Salles ARÉVALO ${ }^{1}$, Tarso da Costa ALVIM ${ }^{1}$
}

\begin{abstract}
Resumo
O presente trabalho de pesquisa teve como objetivo desenvolver um processo de obtenção de pasta de pequi (Caryocar brasiliense) para uso culinário e avaliar a sua estabilidade quando acondicionada em embalagens de plástico e de vidro durante 180 dias de armazenamento. O processamento da pasta envolveu: descascamento, retirada da polpa em forma de lascas, obtenção da pasta em liquidificador, acidificação do produto com ácido cítrico até um $\mathrm{pH} \leq 4,5$ e adição de $10 \%$ de $\mathrm{NaCl}$ (para evitar o desenvolvimento de microrganismos deterioradores e o escurecimento enzimático), tratamento térmico convencional a $80^{\circ} \mathrm{C}$ durante 10 minutos e enchimento a quente em potes de vidro e de plástico com capacidade de $200 \mathrm{~mL}$. O produto final foi armazenado à temperatura ambiente $\left( \pm 28^{\circ} \mathrm{C}\right)$ e submetido a análises físicoquímicas de $\mathrm{pH}$ e acidez e a análises microbiológicas de contagem de fungos e leveduras, coliformes totais e termotolerantes durante um período de 180 dias. Quanto ao tipo de embalagem utilizada, o vidro mostrou-se mais eficiente que a embalagem plástica na proteção contra deterioração microbiana e físico-química, da perda de cor da pasta. Nas embalagens plásticas, após os 60 dias de armazenamento, as pastas apresentavam-se bastante escurecidas, entretanto, nas embaladas de vidro não foi constatada nenhuma evidência de deterioração, apresentando-se em bom estado durante os 120 dias de armazenamento. Para as pastas acondicionadas em vidro, foi observada também uma diminuição do $\mathrm{pH}$ e um aumento da acidez durante o armazenamento. Do ponto de vista microbiológico e físico-químico, a pasta de pequi acondicionada em embalagem de vidro manteve-se estável durante 120 dias, à temperatura ambiente.
\end{abstract}

Palavras-chave: pequi; Caryocar brasiliense; pasta; processamento; estabilidade.

\begin{abstract}
The aim of this paper was to develop a process to obtain a salted and unsalted pequi paste for culinary use and to evaluate its stability when packed in plastic or in glass jars during 180 days of storage. The process included peeling of pequi fruit, pulp cutting, obtain the paste with the use of a blender, acidification of the product with citric acid at a $\mathrm{pH} \leq 4,5$, addition $10 \%$ of $\mathrm{NaCl}$ (to avoid the development of deteriorating microorganisms and enzymatic darkening), thermal treatment at $80{ }^{\circ} \mathrm{C}$ during 10 minutes, and hot filling in plastic and glass jars. The final product was submitted to microbiological and physicochemical analysis of the $\mathrm{pH}$ values and acidity and color evaluation. The results showed what the acidification with citric acid and hot filling process was effective to establish commercial sterility to the pequi paste in glass jars during a four-month period. The paste was more stable when packed in glass jars than in plastic jars with respect to microbiological and physicochemical analysis and color degradation during the four-month period.

Keywords: pequi; Caryocar brasiliense; paste; processing; stability.
\end{abstract}

\section{Introdução}

O cerrado brasileiro apresenta uma rica biodiversidade em alimentos de origem vegetal, principalmente frutos com elevado potencial para a alimentação humana, porém, poucos estudos relacionados ao processamento industrial têm sido realizados. Dentre as frutíferas nativas desta região destaca-se o pequi (Caryocar brasiliense Camb), fruto também conhecido como ouro do cerrado, por seu alto valor econômico e nutricional (FIGUEIREDO, R. W.; MAIA; FIGUEIREDO, E. A., 1989). O pequi é encontrado em todo o cerrado brasileiro, com grandes plantações naturais nos Estados de Maranhão, Piauí, Pará, Mato Grosso, Goiás, Minas Gerais e Tocantins (RIBEIRO, 2000). A polpa de cor amarela, devido ao sabor exótico e ao aroma forte e característico é muito apreciada e utilizada, pela população de vários Estados do Norte, Nordeste e Centro-Oeste, como tempero no preparo de arroz, frango, carnes e na fabricação de licores (LIMA et al., 2007).

Sabe-se que a busca dos consumidores por produtos prontos para consumo cresceu substancialmente na última década, incentivando o desenvolvimento de tecnologias que permitam sua fabricação com qualidade (BERBARI et al., 2003). Desta maneira, a transformação da polpa do pequi em pasta pronta para consumo, estável à temperatura ambiente, tanto do ponto de vista microbiológico como da aparência, facilitaria a utilização desta fruta como tempero, principalmente por sua conveniência de uso. 
Nenhuma pesquisa foi encontrada sobre o processamento e estudo da estabilidade de pasta de pequi em diferentes tipos de embalagens.

A pasta de pequi é um produto que contém água, proteína e uma quantidade elevada de lipídios e de carotenoides. Consequentemente, várias reações deteriorativas podem ocorrer durante o processamento e armazenamento do produto como a perda de cor e outras características sensoriais.

Estas reações podem ser as oxidações de lipídios e de carotenoides. Em alimentos com atividade de água de 0,65 a 0,85, a oxidação dos lipídios é mais pronunciada (OZÇELIK; EVRANUZ, 1998). Quanto à perda de cor, vários fatores são responsáveis durante o processamento e o armazenamento de produtos alimentícios. Estes incluem escurecimento enzimático e não enzimático e condições de processamento como $\mathrm{pH}$, acidez, tipo de embalagem e tempo e temperatura de armazenamento (AHMED; SHIVHARE, 2001).

O presente trabalho teve como objetivo estudar o processamento da pasta de pequi e a estabilidade do produto acondicionado em diferentes tipos de embalagens durante o armazenamento à temperatura ambiente.

\section{Material e métodos}

\subsection{Obtenção e processamento da pasta de pequi}

Os frutos maduros de pequi (Caryocar brasiliense Camb) foram obtidos no período da safra (outubro a novembro) de 2006, provenientes da região do município de Palmas.

No laboratório, os frutos foram primeiramente selecionados quanto ao tamanho e aroma, lavados em água corrente e em seguida foram descascados manualmente, com a ajuda de facas de aço inoxidável. Após descascamento, a polpa do pequi foi separada do caroço mediante o corte da polpa, em forma de laminas ou lascas de $0,5 \mathrm{~cm}$ de espessura, com o auxílio de facas de aço inoxidável. A polpa obtida foi branqueada por imersão em água quente à temperatura de $80{ }^{\circ} \mathrm{C}$ durante 5 minutos para inativação das enzimas, principalmente lípases. Após branqueamento, a polpa foi triturada em liquidificador industrial (Siemsen, poli, Brasil) juntamente com água filtrada na proporção de uma parte de água para uma de polpa, até obtenção de material uniforme em forma de pasta. A quantidade de água adicionada na homogeneização foi determinada conforme testes preliminares, sendo suficiente para obter uma pasta padronizada e consistente.

A pasta obtida foi dividida em duas partes iguais, adicionando-se na primeira parte (Formulação F1) ácido cítrico, em quantidade suficiente para se obter um $\mathrm{pH} \leq 4,5$, sendo que a quantidade foi determinada através de curva de titulação de acordo com a metodologia descrita por Berbari et al. (2003). Na segunda parte (Formulação F2), além do ácido cítrico para obter um $\mathrm{pH} \leq 4,5$, foi adicionado o sal cloreto de sódio na proporção de $10 \%$ p/p, seguindo as recomendações de Carbonell-Barrachina et al. (2003) e de Ahmed e Shivhare (2001). Esta quantidade de sal permite também uma boa redução da microflora, não limitando posteriormente o uso da pasta na culinária.
Em seguida, a pasta formulada foi colocada em um tacho aberto e pasteurizada a $90{ }^{\circ} \mathrm{C}$ durante 10 minutos em constante agitação para se obter uma mistura homogênea. Imediatamente após pasteurização, a pasta foi rapidamente embalada a quente $\left(80^{\circ} \mathrm{C}\right)$ nos potes de vidro e de plástico de $200 \mathrm{~mL}$, fechada hermeticamente e armazenada à temperatura ambiente $\left(28 \pm 1{ }^{\circ} \mathrm{C}\right)$. Os potes de plástico utilizados foram os de polipropileno com fecho hermético através de selagem a quente de selo plástico metalizado, e os potes de vidro foram os de tampa em folha de flandres tipo garra e torção.

\subsection{Análises físico-químicas}

Foram realizadas análises físico-químicas na polpa in natura de acordo com o métodos AOAC (ASSOCIATION..., 1980): umidade, utilizando-se estufa a $105^{\circ} \mathrm{C}$ até obter peso constante; conteúdo de lipídios, através do método de Soxhlet; proteínas, pelo método de Kjeldahl com fator de conversão de 6,25; quantidade de vitamina C, por titulação com 2,6 dicloroindofenol; $\mathrm{pH}$, por leitura direta em potenciômetro; a acidez total titulável, por titulação com solução de $\mathrm{NaOH}$ na titulação. $\mathrm{O}$ teor de cinzas foi determinado após calcinação das amostras em mufla a $550^{\circ} \mathrm{C}$ até peso constante conforme o recomendado pelo Instituto Adolfo Lutz (1985). O teor de carboidratos foi determinado através da diferença de $100 \%$ com a soma das quantidades de umidade, cinzas e proteínas. As análises foram realizadas em triplicata.

\subsection{Análises microbiológicas}

Quanto às análises de desenvolvimento de bolores, leveduras, coliformes totais e termotolerantes, estas foram realizadas segundo a metodologia da APHA (VANDERZANT; SPLITTSTOESSER, 1992). As análises foram realizadas em triplicata.

\subsection{Estudo da vida de prateleira da pasta de pequi}

As pastas processadas e embaladas a quente nos dois tipos de embalagens (plástico e vidro) foram submetidas às seguintes análises físico-químicas: $\mathrm{pH}$ e acidez total titulável; análises microbiológicas de contagem de bolores, leveduras, coliformes totais e termotolerantes e finalmente a análise visual direta de perda da cor. A análise visual foi realizada utilizando o método proposto por Bernhardt et al. (1986) para pasta de cebola. As análises foram realizadas em triplicata após o processamento e em intervalos de 30 dias, por um período de 180 dias à temperatura de $28 \pm 2{ }^{\circ} \mathrm{C}$.

\section{Resultados e discussão}

\subsection{Características físico-químicas da polpa de pequi in natura}

A composição físico-química da polpa de pequi obtida é apresentada na Tabela 1. Através desta tabela, pode-se observar que a polpa apresenta $56,8 \%$ de umidade, $3,1 \%$ de proteína, 2,23\% de cinzas, acidez total titulável de $0,04 \%$ e é rica em lipídios $(21,7 \%)$. Estes valores enquadram-se dentro dos resultados encontrados na literatura (SANO; ALMEIDA,1998; RODRIGUES, 2004). 
Verifica-se, também, que o $\mathrm{pH}$ natural da polpa é de 7,36 sendo classificado como alimento de baixa acidez, ou seja, apresenta $\mathrm{pH}$ acima de 4,5 . Portanto, quando utilizada na formulação de alimentos como pastas, por exemplo, estes devem receber um tratamento térmico de esterilização aplicando temperaturas acima de $100{ }^{\circ} \mathrm{C}$ ou a temperaturas próximas à ebulição da água, desde que seja acidificada a um $\mathrm{pH} \leq 4,5$ para evitar o desenvolvimento de Clostridium botulinum. No entanto, o processo de esterilização a temperaturas maiores que $100{ }^{\circ} \mathrm{C}$ poderá afetar a textura, flavour e cor do pequi (SIQUEIRA, 1997), portanto a acidificação até $\mathrm{pH} \leq 4,5$ seguida de pasteurização seria a tecnologia mais apropriada e barata para o processamento da polpa de pequi na forma de pasta.

\subsection{Curva de acidificação da pasta de pequi}

Nas Figuras 1a e b, mostram-se o comportamento da curva de redução de $\mathrm{pH}$ para $100 \mathrm{~g}$ de pasta de pequi das formulações F1 e F2, em função da concentração do ácido cítrico. Pode-se observar que a quantidade de ácido utilizada para atingir $\mathrm{pH}=4,5$ na formulação F1 é de $0,675 \mathrm{~g}$ de ácido. $100 \mathrm{~g}^{-1}$ de produto e para a formulação F2 de $0,300 \mathrm{~g}$ de ácido. $100 \mathrm{~g}^{-1}$ de produto. Nota-se que as necessidades de ácido para abaixar os valores de $\mathrm{pH}$ ao mesmo valor $(\mathrm{pH}=4,5)$ foram diferentes para as duas formulações, sendo duas vezes maior para a formulação F1 (polpa de pequi mais ácido cítrico) do que para a formulação F2 (polpa de pequi, ácido cítrico mais $10 \%$ de sal).

\subsection{Vida de prateleira da pasta de pequi}

\section{Análise visual direta de perda de cor das pastas}

A polpa de pequi apresenta uma cor amarelo intenso brilhante. Esta cor foi adotada como padrão de coloração para comparação de perda desta durante o armazenamento da pasta de pequi. Na Tabela 2, estão apresentados os resultados da avaliação direta da cor das pastas de pequi embaladas em potes de vidro e de plástico durante o armazenamento.

A avaliação visual direta da cor das pastas de pequi acondicionadas nas diferentes embalagens mostrou que o escurecimento varia em função do tipo de embalagem utilizada. Este escurecimento acontece mais rapidamente nos produtos embalados em potes plásticos, independente do tipo de formulação. Nas embalagens plásticas, após os 60 dias de armazenamento, as pastas apresentavam-se bastante escurecidas e com cheiro característico de ranço, tornando o produto inaceitável do ponto de vista comercial. Entretanto, nas pastas

Tabela 1. Composição físico-química da polpa de pequi in natura utilizado.

\begin{tabular}{lc}
\hline \multicolumn{1}{c}{ Análises } & Resultados \\
\hline Umidade (\%) & 56,80 \\
Lipídios (\%) & 21,70 \\
Proteínas (\%) & 3,10 \\
Cinzas (\%) & 2,23 \\
Carboidratos totais (\%) & 16,17 \\
Vitamina C (mg em 100 g do fruto) & 78,1 \\
Acidez total titulável (\% em ácido cítrico) & 0,042 \\
pH & 7,36 \\
\hline
\end{tabular}

acondicionadas nos potes de vidro, independente da formulação, não foi constatada nenhuma evidência de deterioração, apresentando-se em bom estado durante os cinco meses de armazenamento. No sexto mês, apresentaram uma leve perda de coloração, apenas na superfície do produto, passando do amarelo intenso a um amarelo levemente esbranquiçado, porém sem depreciá-lo após os seis meses de armazenamento. Esta

Tabela 2. Avaliação direta da cor das pastas de pequi embaladas em potes de vidro e de plástico durante o armazenamento.

\begin{tabular}{cccc}
\hline Formulação & Dias & \multicolumn{2}{c}{ Tipo de embalagem } \\
\cline { 3 - 4 } & & Vidro & Plástico \\
\hline F1 & 0 & $\mathrm{~N}$ & $\mathrm{~N}$ \\
& 30 & $\mathrm{~N}$ & $\mathrm{EL}$ \\
& 60 & $\mathrm{~N}$ & $\mathrm{E}$ \\
& 120 & $\mathrm{~N}$ & $\mathrm{E}$ \\
$\mathrm{F} 2$ & 180 & $\mathrm{~N}$ & $\mathrm{E}$ \\
& 0 & $\mathrm{~N}$ & $\mathrm{~N}$ \\
& 30 & $\mathrm{~N}$ & $\mathrm{~N}$ \\
& 60 & $\mathrm{~N}$ & $\mathrm{E}$ \\
& 120 & $\mathrm{~N}$ & $\mathrm{E}$ \\
& 180 & $\mathrm{NE}$ & $\mathrm{E}$ \\
\hline
\end{tabular}

Observação: $\mathrm{N}$ - cor normal (amarelo intenso), NE - cor normal levemente esbranquiçada, EL - escurecido levemente (amarelo para marrom), E - escurecido (marrom escuro).
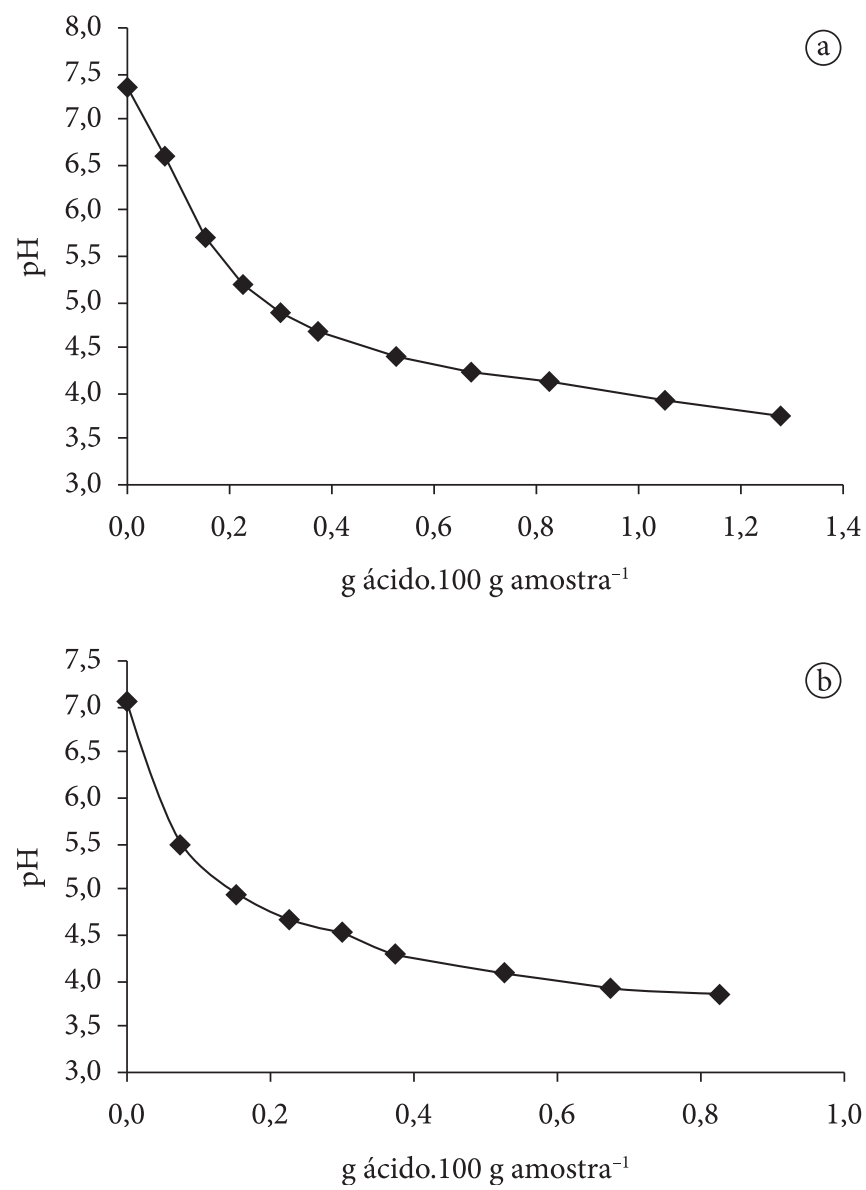

Figura 1. a) Curva de acidificação da formulação F1 e b) formulação F2. 
perda de coloração também foi observada por Ahmed e Shivhare (2001), trabalhando com pastas de cebola embaladas em sacos plásticos de polietileno e armazenadas às temperaturas de $5 \mathrm{e}$ $25{ }^{\circ} \mathrm{C}$ durante 60 dias. Fato semelhante também foi observado por Carbonell-Barrachina et al. (2003) durante o processamento de pasta de alho acidificado a $\mathrm{pH} \leq 4,5$, adicionado de $10 \%$ de $\mathrm{NaCl}$ e embalado em potes de plásticos.

A polpa de pequi é um produto gorduroso e com alto teor de carotenoides, que lhe dão a coloração característica de amarelo intenso, desta forma, as pastas de pequi são bastante susceptíveis a reações de oxidação dos ácidos graxos da gordura e dos carotenoides com perda de coloração das pastas. Segundo Garcia et al. (1989), a oxidação destes componentes são acelerados por fatores como luz, calor e oxigênio. Portanto, essa oxidação dos ácidos graxos e dos carotenoides está diretamente relacionada com a embalagem utilizada, ou seja, enquanto o vidro é impermeável à passagem de gases e vapores, o polipropileno não apresenta alta barreira, mas apresenta maior resistência à passagem da luz que o vidro, que é totalmente transparente.

Como a cor e a aparência do produto são os primeiros fatores a chamar a atenção do consumidor e como ele nunca provará a pasta de pequi diretamente, consideram-se esses parâmetros como um dos mais importantes na avaliação da vida útil para comercialização do produto (SAENZ et al. 1993; AHMED; SHIVHARE, 2001).

\section{Análises microbiológicas}

Na Tabela 3, encontram-se os resultados das análises microbiológicas da pasta de pequi embaladas nos potes de vidro e de plástico durante o armazenamento a temperatura ambiente. Através desta Tabela pode-se notar que todas as pastas acondicionadas em potes de vidro permaneceram estáveis microbiologicamente, apresentando a contagem de coliformes totais e termotolerantes e de bolores e leveduras abaixo dos padrões microbiológicos estabelecidos pela legislação brasileira, de $10^{4} \mathrm{ufc} \cdot \mathrm{g}^{-1}$ para os produtos prontos para o consumo (AGÊNCIA...,, 2001). A acidificação das pastas nos níveis de $\mathrm{pH} \leq 4,5$, acompanhada de adição do sal e aquecimento a $90{ }^{\circ} \mathrm{C}$ com a consequente embalagem a quente e fechamento hermético, foi suficiente para reduzir a flora microbiana, conferindo estabilidade ao armazenamento por 6 meses, para os produtos embalados em potes de vidro. Entretanto, para as pastas embaladas nos potes de plástico, pode-se verificar que além do escurecimento do produto, como comentado anteriormente, foi constatado o crescimento microbiano acima do permitido pela legislação, na formulação F2 após os 30 dias de armazenamento e valores muito próximos para F1. Nota-se, também, que os bolores e leveduras crescem mais lentamente na formulação F2 que contém na sua composição $10 \%$ de sal, havendo uma redução no crescimento aos 180 dias de armazenamento. Segundo Bozkurt e Erkmen (2004) a adição de sal ajuda a diminuir ou a reduzir a flora microbiana sobre a pasta, por inibição própria e por reduzir a atividade de água no produto.

Os coliformes fecais são micro-organismos que comprometem a qualidade sanitária dos alimentos, são indicadores de contaminação fecal do produto e, portanto, do risco de transmissão de patógenos presentes nas fezes (FORSYTHE, 2002). $\mathrm{Na}$ presente pesquisa, foi constatada a ausência de coliformes no produto, observando que houve observância de práticas de higiene durante todo o processamento.

\section{Análises físico-químicas}

As análises físico-químicas realizadas nas pastas de pequi acondicionadas nos dois tipos de embalagens durante o armazenamento estão apresentadas na Tabela 4. Para as pastas embaladas em potes plásticos, apenas foram realizadas análises durante os primeiros 30 dias após o processamento devido

Tabela 4. Valores de $\mathrm{pH}$ e acidez (g ácido cítrico. $100 \mathrm{~g}^{-1}$ ) durante o armazenamento de pastas de pequi embaladas em potes de vidro e de plástico.

\begin{tabular}{ccccccc}
\hline Formulação & Dias & \multicolumn{3}{c}{$\mathrm{pH}$} & & \multicolumn{2}{c}{ Acidez $^{*}$} \\
\cline { 3 - 4 } \cline { 6 - 7 } & & Vidro & Plástico & & Vidro & Plástico \\
\hline F1 & 0 & 4,57 & 4,57 & & 0,30 & 0,27 \\
& 30 & 4,34 & 4,37 & & 0,33 & 0,22 \\
& 60 & 4,37 & $\mathrm{~d}$ & & 0,39 & $\mathrm{~d}$ \\
& 120 & 4,21 & $\mathrm{~d}$ & & 0,39 & $\mathrm{~d}$ \\
& 180 & 4,16 & $\mathrm{~d}$ & & 0,39 & $\mathrm{~d}$ \\
$\mathrm{~F} 2$ & 0 & 4,53 & 4,71 & & 0,22 & 0,24 \\
& 30 & 4,64 & 5,67 & & 0,21 & 0,30 \\
& 60 & 4,65 & $\mathrm{~d}$ & & 0,30 & $\mathrm{~d}$ \\
& 120 & 4,46 & $\mathrm{~d}$ & & 0,29 & $\mathrm{~d}$ \\
& 180 & 4,39 & $\mathrm{~d}$ & 0,32 & $\mathrm{~d}$ \\
\hline
\end{tabular}

*\% em ácido cítrico; $\mathrm{d}$ - deteriorado.

Tabela 3. Análises microbiológicas durante o armazenamento de pastas de pequi embaladas em potes de vidro e plástico.

\begin{tabular}{|c|c|c|c|c|c|c|c|}
\hline \multirow[t]{2}{*}{ Formulação } & \multirow[t]{2}{*}{ Dias } & \multicolumn{2}{|c|}{ Coliformes totais (NMP.g ${ }^{-1}$ ) } & \multicolumn{2}{|c|}{ Coliformes termotolerantes (NMP.g ${ }^{-1}$ ) } & \multicolumn{2}{|c|}{ Bolores e leveduras (UFC.g ${ }^{-1}$ ) } \\
\hline & & Vidro & Plástico & Vidro & Plástico & Vidro & Plástico \\
\hline \multirow[t]{4}{*}{ F1 } & 0 & $<10^{3}$ & $<10^{3}$ & $<10^{3}$ & $<10^{3}$ & $<10$ & $7,0 \times 10^{3}$ \\
\hline & 30 & $<10^{3}$ & $<10^{3}$ & $<10^{3}$ & $<10^{3}$ & $<10$ & $1,5 \times 10^{3}$ \\
\hline & 120 & $<10^{3}$ & $\mathrm{~d}$ & $<10^{3}$ & $\mathrm{~d}$ & $8,0 \times 10^{3}$ & $\mathrm{~d}$ \\
\hline & 180 & $<10^{3}$ & $\mathrm{~d}$ & $<10^{3}$ & $\mathrm{~d}$ & $8,8 \times 10^{3}$ & $\mathrm{~d}$ \\
\hline \multirow{3}{*}{$\mathrm{F} 2$} & 60 & $<10^{3}$ & $\mathrm{~d}$ & $<10^{3}$ & $\mathrm{~d}$ & $6,0 \times 10^{2}$ & $\mathrm{~d}$ \\
\hline & 120 & $<10^{3}$ & $\mathrm{~d}$ & $<10^{3}$ & $\mathrm{~d}$ & $7,0 \times 10^{3}$ & $\mathrm{~d}$ \\
\hline & 180 & $<10^{3}$ & $\mathrm{~d}$ & $<10^{3}$ & $\mathrm{~d}$ & $3,0 \times 10^{3}$ & d \\
\hline
\end{tabular}

d - deteriorado 
à perda de coloração. Os resultados são a média das análises realizadas em duplicata.

Observa-se que existe uma tendência de aumento da acidez e uma diminuição do $\mathrm{pH}$ em todos os produtos durante o armazenamento. Para as pastas embaladas em vidro, o valor de $\mathrm{pH}$ decresce gradualmente durante todo o período de armazenamento, sendo este decréscimo muito maior na pasta F1 (4,57 a 4,16) que na F2 (4,6 a 4,39), indicando que a adição de $10 \%$ sal ajudou a prevenir o incremento do $\mathrm{pH}$, principalmente pelo controle microbiano que o sal oferece. Similares observações foram reportadas por Gamli e Hayoglu (2007) durante o armazenamento de pasta de pistachio, um produto altamente gorduroso e por Bozkurt e Erkmen (2004) durante o armazenamento de pasta de pimentão formulada com $5 \%$ de sal e embalada em sacos plásticos.

A acidez das pastas acondicionadas em embalagens de plástico durante os 30 dias de observação variou de 0,27 a 0,22 para a F1 sem sal e de 0,24 a 0,30 para F2 formulada com $10 \%$ de sal. Para as pastas embaladas em vidro, a acidez variou de 0,30 a 0,39 para F1 e de 0,22 a 0,32 para F2. Estes resultados estão dentro da faixa de acidez encontrados por Ahmed e Shivhare (2001) para pasta de cebola. Quanto à acidez das pastas embaladas em vidro, nota-se um aumento durante todo o período de armazenamento, para a formulação F2. Porém, para F1, este aumento foi apenas notado durante os primeiros 60 dias de armazenamento, quando então permanece constante em 0,39 . Os valores de acidez aumentam de 0,30 a 0,39 para F1 e de 0,22 a 0,32 para F2, verificando-se que a adição de $10 \%$ de sal na formulação F2 não influenciou na acidez. Este aumento da acidez em todas as formulações indica o início de reações hidrolíticas dos ácidos graxos presentes em altas concentrações na pasta de pequi. Gamli e Hayoglu (2007), estudando o efeito do tipo de embalagem (vidro e plástico) e temperatura de armazenamento sobre a qualidade da pasta de amêndoa de pistachio, também verificaram essa tendência de aumento da acidez. Fato semelhante foi observado por Torun (1999), durante a avaliação da estabilidade de pasta de nozes durante o armazenamento.

\section{Conclusões}

O enchimento a quente em potes de vidro em conjunto com um processo de acidificação eficaz é capaz de manter estável a pasta de pequi por um período de pelo menos 120 dias.

As embalagens plásticas de polipropileno utilizadas neste estudo não se mostraram viáveis para o produto, visto que ocorreu uma rápida perda de coloração que deprecia comercialmente o produto.

Em termos microbiológicos e físico-químicos, todas as amostras acondicionadas em embalagens de vidro, até o período de 120 dias de armazenamento, permaneceram praticamente inalteradas dentro dos padrões estabelecidos pela legislação, demonstrando que o processamento efetuado para a obtenção da pasta de pequi é efetivo no combate aos microrganismos deteriorantes e patogênicos.

\section{Referências bibliográficas}

AGÊNCIA NACIONAL DE VIGILÂNCIA SANITÁRIA - ANVISA. Resolução RDC n 12, de 2 de janeiro de 2001. Diário Oficial da União, 10 de janeiro de 2001.
AHMED, J.; SHIVHARE, U. S. Thermal of color degradation and storage characteristics of onion paste. Lebensmittel- Wissenschaft und- Technologie, v. 34, p. 380-383, 2001.

ASSOCIATION OF OFFICIAL ANALITICAL CHEMISTS - AOAC. Official Methods of Analysis. [S.1.], 1980. 200 p.

BERBARI, S. A. G.; SILVEIRA, N. F. A.; OLIVEIRA, L. A. T. Avaliação do comportamento de pasta de alho durante o armazenamento (Allium sativum L.). Ciência e Tecnologia de Alimentos, v. 23, n. 3, p.468-472, 2003.

BERNHARDT, L. W. et al. Processamento de pasta de cebola e sua estabilidade em diferentes embalagens. Coletânea do Instituto de Tecnologia de Alimentos, v. 23, n. 1, p. 87-102, 1986.

BOZKURT, H.; ERKMEN, O. Effects of production techniques on the quality of hot pepper paste. Journal of Food Engineering, v. 64, p 173-178, 2004.

CARBONELL-BARRACHINA, A. A. et al. Development of a High Sensory Quality Garlic Paste. Journal of Food Science, v. 68, n. 7, p. 2351-2355, 2003.

FIGUEIREDO, R. W.; MAIA, G. A.; FIGUEIREDO, E. A. Propriedades físico-químicas e composição dos ácidos graxos da fração lípidica da polpa e amêndoa do pequi (Caryocar coriaceum Wittm). Ciências Agronômicas, v. 20, n. 1/2, 1989.

FORSYTHE, S. J. Microbiologia da Segurança Alimentar. São Paulo: Artmed, 2002. 424 p.

GAMLI, O. F.; HAYOGLU I. The effect of the different packaging and storage conditions on the quality of pistachio nut paste. Journal of Food Engineering, v. 78, p. 443-448, 2007.

GARCIA, P. et al. Color and texture of acidified ripe olives in pouches. Journal of Food Science, v. 50, p. 1115-1129, 1989.

INSTITUTO ADOLFO LUTZ - IAL. Normas Analíticas do Instituto Adolfo Lutz. Métodos Químicos e Físicos para Análise de Alimentos. 3. ed. São Paulo: IAL, 1985. 533 p.

LIMA, A. et al. Composição química e compostos bioativos presentes na polpa e na amêndoa do pequi (Caryocar brasiliense). Revista Brasileira Fruticultura, v. 29, n. 3, p. 695-698, 2007.

OZÇELIK, B.; EVRANUZ, O. Lipid oxidation in oil seed: Effective factors and measurement methods. Food Technology, v. 23, n. 3, p. 221-227, 1998.

RIBEIRO, R. F. Pequi o rei do cerrado. Belo Horizonte: Rede Cerrado, 2000. p. 62.

RODRIGUES, L. J. O Pequi (Caryocar brasiliense Camb): ciclo vital e agregação de valor pelo processamento mínimo. $150 \mathrm{f}$. Dissertação (Mestrado em Ciência dos Alimentos)-Universidade Federal de Lavras, Lavras, 2004.

SAENZ, C. et al. Color changes in concentrated juices of princkly pear (Opuntia ficus indica) during storage at different temperatures. Lebensmittel- Wissenschaft und- Technologie, v. 26, p. 417-421, 1993.

SANO, S. M.; ALMEIDA, S. P. Cerrado: ambiente e flora. Planaltina: EMBRAPA-CPAC, 1998. 556 p.

SIQUEIRA, M. I. D. Conserva de pequi. Goiânia: [s.n.], 1997. 22 p. (Manual Técnico, 2).

TORUN, B. The production methods of walnut paste, improving the shelf life and quality. Antalya: Institute of Natural and Applied Science, University of Mediterranean, 1999. $15 \mathrm{p}$.

VANDERZANT, T.; SPLITTSTOESSER, E. F. Compendium of methods for the microbiological examination of foods. 3. ed. Washington: American Public Health Association - APHA, 1992. 1919 p. 